\title{
Experimental observation of edge states in SSH-Stub photonic lattices
}

\author{
Gabriel Cáceres-Aravena, ${ }^{1,2}$ Bastián Real $\odot,{ }^{3}$ Diego Guzmán-Silva, ${ }^{1,2}$ Alberto Amo $\odot,{ }^{3}$ Luis E. F. Foa Torres $\odot,{ }^{1}$ \\ and Rodrigo A. Vicencio (1) 1,2 \\ ${ }^{1}$ Departamento de Física, Facultad de Ciencias Físicas y Matemáticas, Universidad de Chile, Santiago 8370448, Chile \\ ${ }^{2}$ Millenium Institute for Research in Optics - MIRO, Universidad de Chile, Santiago 4130691, Chile \\ ${ }^{3}$ Univ. Lille, CNRS, UMR 8523-PhLAM-Physique des lasers Atomes et Molécules, F-59000 Lille, France
}

(Received 28 October 2021; accepted 28 January 2022; published 7 March 2022)

\begin{abstract}
We reveal unconventional edge states in a one-dimensional Stub lattice of coupled waveguides with staggered hoppings. The edge states appear for the same values of hoppings as topological edge states in the Su-SchriefferHeeger model. They have different energies depending on the lattice termination and present a remarkable robustness against certain types of disorder. We evidence experimentally the phase transition at which these edge states appear, opening the door to the engineering of one-dimensional lattices with localized edge modes whose energy and location can be controlled at will.
\end{abstract}

DOI: 10.1103/PhysRevResearch.4.013185

\section{INTRODUCTION}

During the last few years, there has been a surge of interest in the study of topological edge states in a plethora of systems, ranging from electronic states in materials [1] to analogs in mechanical [2] and photonic settings [3,4]. In particular, the field of topological photonics [3,4] has bloomed over the last decade. But beyond the much sought-for topological protection, a few studies [5-8] have shown that sometimes an enhanced robustness (and even antifragile behavior) can be achieved by breaking the rules and tinkering with edge states outside the topological phase. This includes, for example, edge states coexisting with a gapless bulk [5], states of metals on topological insulator systems [6], and also many Floquet systems $[7,8]$ which are effectively gapless but which nevertheless show robust edge states.

In one dimension, topological phases require imposing a symmetry, typically chiral or sublattice symmetry, as in the case of the Su-Schrieffer-Heeger (SSH) model $[9,10]$. The resulting topological phase has edge states which, notwithstanding their "topological protection," are not as strong as their two-dimensional counterpart. Furthermore, the peculiar status of chiral symmetry, which is different from usual crystalline symmetries, brings more subtleties to the onedimensional case. Thus, one may wonder whether when going beyond the constraints of topology one could find edge states equally robust to those of the paradigmatic SSH model. Such a scheme would have the potential to provide more useful edge states for diverse applications in physics [4].

Published by the American Physical Society under the terms of the Creative Commons Attribution 4.0 International license. Further distribution of this work must maintain attribution to the author $(s)$ and the published article's title, journal citation, and DOI.
Here, we study a modified version of the SSH lattice, that we call the SSH-Stub model. We show that this model sustains edge states with unusual properties. On one hand, there is an imbalanced number of edge states at the two ends of the chain, and they have different energies: Two are located at one edge with nonzero energy, and one midgap state is present at the opposite edge. On the other hand, since the model has a net chiral charge [11] the midgap state coexists with flatband modes. Furthermore, the edge states enjoy a robustness comparable to those found in the SSH model; therefore, our results open a path for robust edge states beyond the standard topological classification.

\section{SSH-STUB MODEL}

The propagation of light on a dimerized Stub photonic lattice, which is composed of sites $A, B$, and $C$ as sketched in Fig. 1(a), is well described by a tight-binding-like model [12,13] as follows,

$$
-i \frac{d u_{n}}{d z}=\sum_{m \neq n} t_{n, m} u_{m}
$$

Here, $u_{n}$ is the light amplitude of the fundamental mode at the $n$th waveguide, $z$ the propagation direction (dynamical variable), and $t_{n, m}$ is the coupling (hopping) coefficient between sites $n$ and $m$ (the distance in between sites $A, B$, and $C$ determines the strength of these coefficients). We identify the coupling constants $t_{2}$ and $t_{3}$ as the horizontal and vertical intracell couplings, respectively, while $t_{1}$ defines the intercell horizontal coupling. Depending on their values, the system experiences different regimes, which we characterize using the parameter $\delta \equiv t_{2} / t_{1}$. Bands are obtained using a Bloch ansatz directly in model (1), obtaining

$$
\beta_{z}=0, \quad \beta_{z}^{ \pm} \equiv \pm \sqrt{\left(1+2 \delta \cos k+\delta^{2}\right) t_{1}^{2}+t_{3}^{2}}
$$



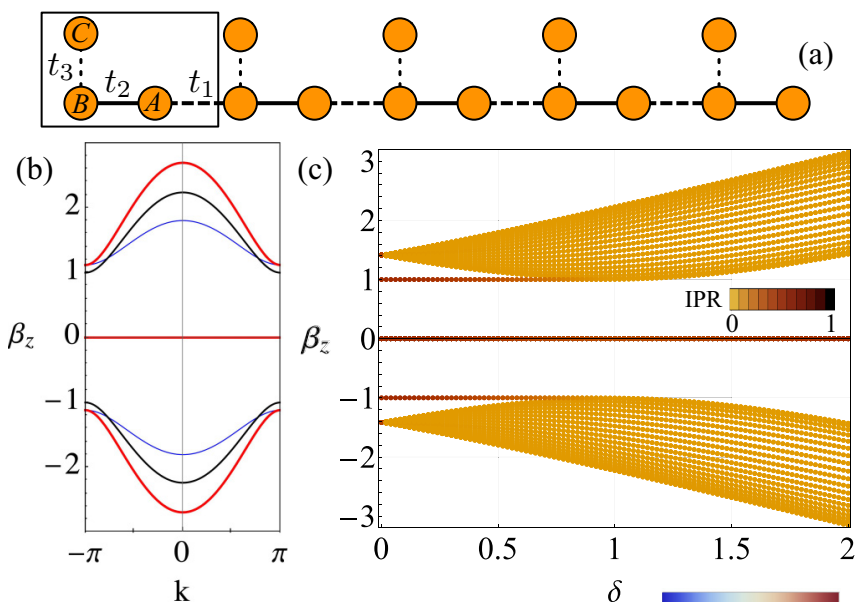

$\beta_{z}$
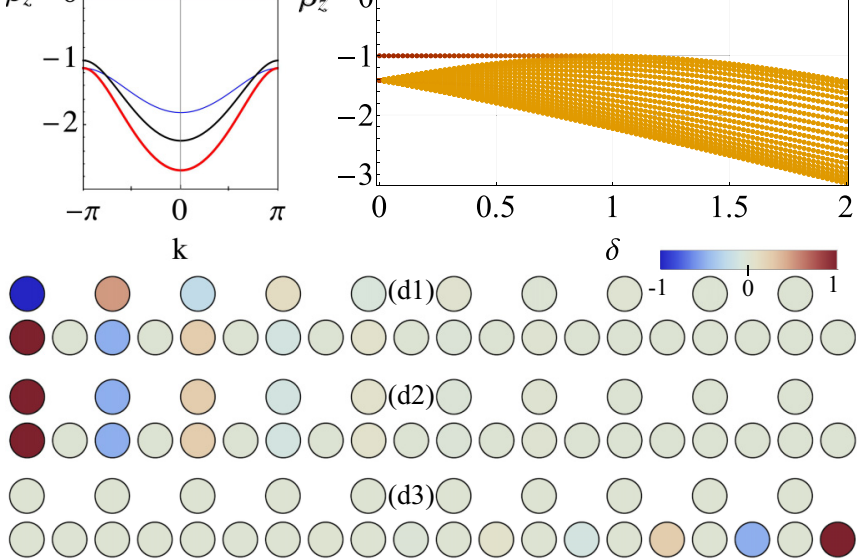

FIG. 1. (a) Scheme of a finite SSH-Stub lattice. The unit cell is marked with a rectangle. (b) Dispersion relation of an infinite lattice for $\delta=0.5$ (blue), $\delta=1.0$ (black), and $\delta=1.5$ (red). The FB at $\beta_{z}=$ 0 exists for any value of $\delta$. (c) Spectrum for different values of $\delta$. (d1) $-(\mathrm{d} 3)$ Amplitude profiles of left $\left(\beta_{z}= \pm 1\right)$ and right $\left(\beta_{z}=0\right)$ edge states. In (b) and (c) $t_{1}=t_{3}=1$.

We plot them in Fig. 1(b) for three different values of $\delta$, where $k$ defines the transversal quasimomentum. The spectrum is composed by a flat band (FB) [14] at $\beta_{z}=0$, and two dispersive bands $\beta_{z}^{ \pm}$which have a symmetric spectrum, where each positive frequency is paired to a negative one [15]. The coupling parameter $t_{3}$ determines the size of dispersive bands and the gap in between bands $\beta_{z}^{ \pm}$. FB modes consist of only three lattice sites: two consecutive $C$ and one $A$ amplitudes, with null $B$ sites [16]. Now, by considering a finite lattice with boundaries as the ones shown in Fig. 1(a), we numerically compute the spectrum for different $\delta$ values [see Fig. 1(c)]. We clearly observe the flat band at $\beta_{z}=0$ and also the two symmetric dispersive bands $\beta_{ \pm}$. Interestingly, two new states emerge in the internal gap of the dispersive bands for $|\delta|<1$, which we identify as edge states with $\beta_{z}= \pm t_{3}$. Figures $1(\mathrm{~d} 1)$ and $1(\mathrm{~d} 2)$ show their spatial profiles: They present a zero amplitude at $A$ sites, and an exponential decay towards the bulk for $B$ and $C$ sites. The high-energy state at $\beta_{z}=t_{3}$ has a bonding phase structure within each unit cell, while the low-energy one at $\beta_{z}=-t_{3}$ has an antibonding character. By inspecting the opposite (right) edge, which ends with an $A$ site, we notice two extra edge modes at $\beta_{z}=0$. One of them is trivial and it corresponds to a truncated FB state that exists for any value of $\delta$. A second one is a hidden $(h)$ edge mode, which exists for $|\delta|<1$ and decays exponentially into the bulk with nonzero amplitude only on $A$ sites. Notice that there is an imbalanced number of edge states on the boundaries due to the coupling dimerization: Two edge states appear at the left boundary and one edge state exists at the right boundary. This fact differs from other topological models such as the SSH lattice [9], in which the same number of edge modes appear at both boundaries.

The localization properties are studied by using an standard definition of the inverse participation ratio, IPR $=$ $\sum_{n}\left|u_{n}\right|^{4} /\left(\sum_{n}\left|u_{n}\right|^{2}\right)^{2}$. IPR $\rightarrow 1[1 /(3 N)]$ for a localized (delocalized) profile, with $N$ the number of unit cells. Figure 1(c) shows that the bulk $\beta_{z}^{ \pm}$states are extended having a small IPR, whereas the FB localized modes possess a high IPR value. This figure also shows that the edge states at $\beta= \pm t_{3}$ are well localized for $\delta \rightarrow 0$, while the IPR decreases for $\delta \rightarrow 1$. These edge modes can be analytically expressed as follows,

$$
\left\{B_{n}^{e}(z), C_{n}^{e}(z)\right\}=\left\{B_{e}, C_{e}\right\} \epsilon^{n-1} \exp \left(i \beta_{z}^{e} z\right), \quad A_{n}^{e}(z)=0,
$$

with $n \geqslant 1$ (starting at the left boundary) and considering $|\epsilon|<1$, such that the profile decays exponentially from the surface into the bulk. We find that $C_{e}= \pm B_{e}, \epsilon=-\delta$, and $\beta_{z}^{e}= \pm t_{3}$, corroborating the existence condition $|\delta|<1$. The IPR of these edge states can be analytically written as $\mathrm{IPR}_{e}=$ $\left(1-\delta^{2}\right) /\left[2\left(1+\delta^{2}\right)\right]$. Therefore, $\mathrm{IPR}_{e} \rightarrow 1 / 2$ for $\delta \rightarrow 0$, as only the $B$ and $C$ sites of the first unit cell have a nonzero amplitude. $\mathrm{IPR}_{e} \rightarrow 0$ for $\delta \rightarrow 1$, evidencing that the edge states bifurcate from completely delocalized bulk states. Furthermore, we analytically construct the hidden edge state as

$$
A_{n}^{h}(z)=A_{N} \gamma^{N-i} \exp \left(i \beta_{z}^{h} z\right), \quad B_{n}(z)=C_{n}(z)=0,
$$

for $n \leqslant N$. We find that $\gamma=-\delta$ and $\beta_{z}^{h}=0$ for $|\delta|<1$, which is the same existence condition of the two previous edge states. This state has a $\mathrm{IPR}_{h}=2 \mathrm{IPR}_{e}$ because only $A$ sites are excited. $\mathrm{IPR}_{h} \rightarrow 1$ for $\delta \rightarrow 0$ (maximal localization) and $\mathrm{IPR}_{h} \rightarrow 0$ for $\delta \rightarrow 1$, which indicates that this edge state mixes with completely delocalized states composed of a superposition of FB states. It is possible to reveal this edge state, for example, by adding an on-site energy at the $A$ sites and destroying the flat band.

The existence condition $|\delta|<1$ for the three exponentially decaying edge modes suggests a close relation between our model and the SSH one $[9,17]$. Our model possesses a unit cell formed by three sites, therefore we expect to find three edge states only. The third state is equivalent to the SSH one, due to the fact both $B$ and $C$ amplitudes are zero. However, the left-hand edge modes are naturally detuned in energy to $\beta_{z}= \pm t_{3}$. They exist as vertical dimers coupled by $A$ null sites following an SSH dimerization. Although our edge modes show similarities with the topologically nontrivial edge states of an SSH system, including the chiral symmetry [18], we cannot assign them a topological origin due to the absence of a well-defined topological invariant [19].

\section{EXPERIMENTAL DEMONSTRATION OF THE EDGE STATES}

We use a photonic platform to verify experimentally the existence of edge states on SSH-Stub lattices. Optical waveguide arrays are fabricated using a femtosecond laser writing technique [20], as sketched in Fig. 2(a). Ultrashort pulses (red beam in the figure) from a Yb-doped fiber laser (Menlo Systems BlueCut, wavelength of $1030 \mathrm{~nm}$, repetition rate of $500 \mathrm{kHz}$, pulse width $\sim 230 \mathrm{fs}$ ) are tightly focused inside a borosilicate Eagle XG glass wafer (yellow block in the 


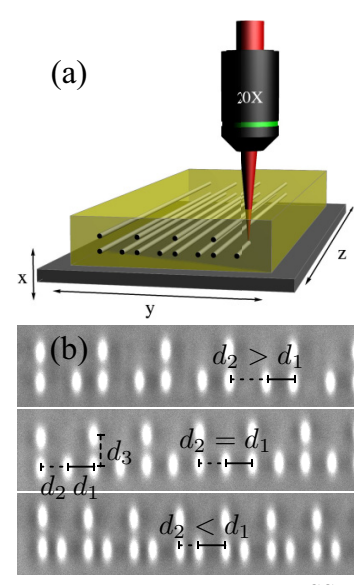

(c)

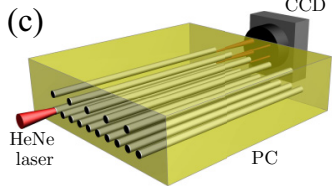

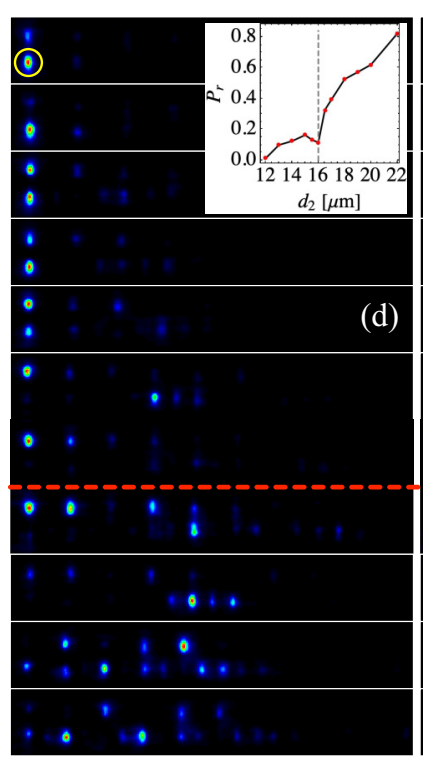
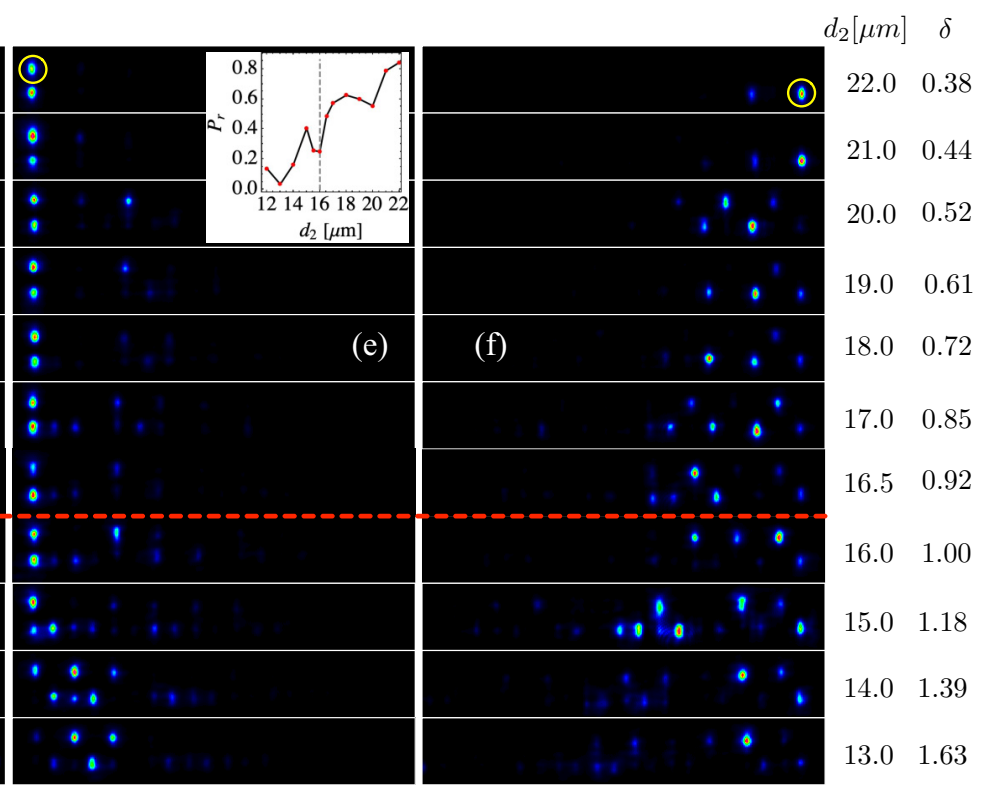

FIG. 2. (a) Femtosecond laser writing technique. (b) Bright-field microscopy of dimerized Stub photonic lattices for $d_{1}=16 \mu \mathrm{m}, d_{3}=$ $18 \mu \mathrm{m}$, and $d_{2}=22$ (top), 16 (center), and 12 (bottom) $\mu \mathrm{m}$, respectively. (c) HeNe characterization setup. (d)-(f) Intensity output profiles for edge- $B$, edge- $C$, and edge- $A$ excitations (see yellow circle), respectively. $d_{2}$ and $\delta$ are indicated directly in the figure. A dashed horizontal line indicates the transition region. Insets in (d) and (e): $P_{r}$ vs distance $d_{2}$.

figure) by using a $20 \times$ microscope objective. Then, samples are continuously translated using a motorized Thorlabs XYZ stage (gray plate in the figure), thus creating waveguides inside the sample (see cylinders inside the glass), achieving a refractive index contrast $\Delta n \sim 10^{-4}-10^{-3}$, on a borosilicate material with a nominal refractive index of $n_{0}=1.48$. The writing velocity was set to $v=0.4 \mathrm{~mm} / \mathrm{s}$ and kept fixed along this experiment. The pulse energy used for all waveguides was of $0.16 \mu \mathrm{J}$ ( $\sim 80 \mathrm{~mW}$ average power), implying singlemode operation at $633 \mathrm{~nm}$. The propagation length for all the waveguides corresponds to the total length of the sample, $L=5 \mathrm{~cm}$. Every waveguide has an elliptical transversal shape, of approximately $4 \times 11 \mu \mathrm{m}$. Figure 2(b) shows three different lattices after illumination, where the dimerization is clearly revealed as well as the waveguide ellipticity. As the excitation of edge states is determined by the parameter $\delta$, it is mandatory to dimerize the coupling interaction in between sites $A$ and $B$. The dimerization is experimentally implemented by varying the intracell distance $d_{2}$, while keeping constant the intercell $\left(d_{1}=16 \mu \mathrm{m}\right)$ and vertical $\left(d_{3}=\right.$ $18 \mu \mathrm{m}$ ) distances, considering that coupling constants decay exponentially with the distance [20] [in our experiment, coupling constants were fit as $t_{h}(d)=15.41 \exp \{-0.1631 d\}$ and $\left.t_{v}(d)=53.64 \exp \{-0.2139 d\}\right]$. Light from a HeNe laser at $633 \mathrm{~nm}$ was tightly focused by a $10 \times$ microscope objective at the input facet of a photonic chip (PC), as described in Fig. 2(c). This allows us to excite single waveguides only, which is equivalent to excite the lattices using deltalike input conditions in model (1). Near-field intensity images are taken by a CCD camera at the output facet of the PC, after a total propagation length of $5 \mathrm{~cm}$.

First of all, we excite the lattice at a bulk- $B$ site to demonstrate good transport conditions. We notice that, independently of the value of $d_{2}$, a clear light broadening (transport) is observed in all cases. This is due to the fact that bulk- $B$ sites excite dispersive bands only, with a null superposition of the flat band. Then, we excite an edge- $B$ site and collect results in Fig. 2(d). This input condition excites extended propagating modes and edge states, but no FB modes. Therefore, if a localized edge state exists, we may observe the energy trapped at the left-edge region. We observe clearly that a well-localized edge profile is excited for distances $d_{2}>d_{1}$ $(\delta<1)$, with almost no tail nor relevant transport through the lattice. Then, for $d_{2}=d_{1}(\delta=1)$ we observe a kind of broader profile as expected in the limit $\delta \rightarrow 1$. Afterwards, for $d_{2}<d_{1}$ $(\delta>1)$, we do not observe any significant localization in the waveguides close to the edge, which is evidence that no edge states are found at this parameter region. In order to quantify the amount of energy which remains trapped at the left boundary versus the energy in the whole lattice, we define the ratio $P_{r} \equiv\left(\left|B_{1}\right|^{2}+\left|C_{1}\right|^{2}\right) / \sum_{n}\left|u_{n}\right|^{2} \cdot P_{r}=1$ means that all the energy is fully localized at the $B_{1}$ and $C_{1}$ (edge) sites. Figure 2(d) (inset) shows $P_{r}$ vs $d_{2}$, where we observe quite clearly a transition into delocalization while $d_{2}$ decreases. The energy remains localized for $d_{2}>d_{1}$, while it spreads out from the edge for $d_{2}<d_{1}$. This is a direct experimental demonstration of the existence of edge modes for $\delta<1$ at the left boundary, in perfect agreement with our theoretical findings.

Now, we excite an edge- $C$ site and present our results in Fig. 2(e). A $C$ site excites all bands, including the FB and the edge modes as well. Then, for $d_{2}<d_{1}$ we observe a localized pattern where $A$ and $C$ sites are mostly excited. This is clear indication of a FB excitation with a quite localized spatial profile [16]. When $d_{2}>d_{1}$ the light intensity gets localized in the outermost $B$ and $C$ sites, evidencing the excitation of the edge mode. In this case, the observed transition is not as abrupt as when exciting the $B$ site due to the simultaneous 

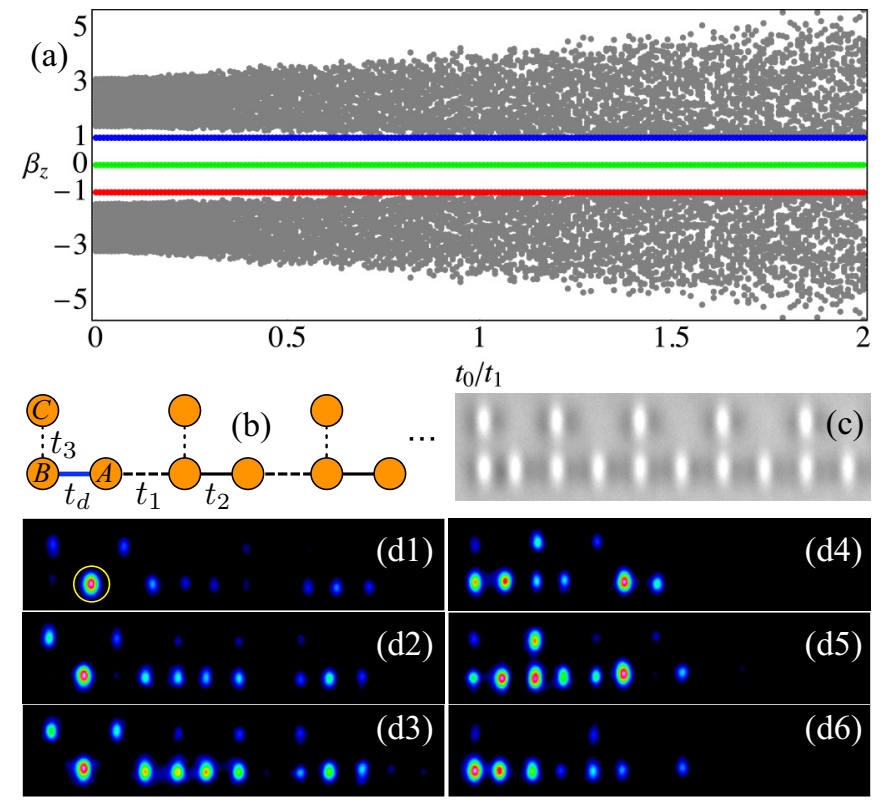

FIG. 3. (a) Averaged spectrum of a disordered SSH-Stub lattice vs the disorder strength $t_{0} / t_{1}$, for $\delta=0.5$. Blue, green, and red dots indicate the energies of the edge states, while gray dots correspond to the energies of bulk states. (b) Scheme for a SSH-Stub lattice including a coupling defect $t_{d}$ at the first unit cell. (c) Microscope image of a SSH-Stub photonic lattice for $\left\{d_{1}, d_{2}, d_{3}, d_{d}\right\}=\{16,14,18,12\} \mu \mathrm{m}$. (d) Intensity output profiles for an $A$-site excitation (see yellow circle). (d1)-(d6) $d_{d}=19,17,16,15,14,12 \mu \mathrm{m}$, respectively.

excitation of localized FB modes. On the other hand, when exciting the $A$ site at the right-hand boundary [see Fig. 2(f), two uppermost panels], we observe the third edge state for $\delta \ll 1$ what is direct proof of the existence of this hidden mode. This excitation strongly overlaps with the FB mode at the right edge while $\delta\left(d_{2}\right)$ increases (decreases), and we observe a clear superposition of FB modes forming a zigzaglike spatial configuration with null $B$ sites. For $d_{2}<d_{1}$, the edge FB amplitude $A_{N}$ decreases and the absence of the third nontrivial edge state is evident by observing the penetration of the energy into the bulk of the lattice. The excited spectrum for all the experimental cases described in Figs. 2(d)-2(f) is addressed in Appendix A, where we analyze the specific excited bands depending on the input excitation.

The robustness of these edge states can be probed numerically adding random off-diagonal disorder into model (1) in the form $t_{1}+t_{0} \epsilon_{1}$ and $t_{2}+t_{0} \epsilon_{2}$, such that every link of the lattice takes a different value ( $t_{0}$ is the disorder strength and $\epsilon_{1,2}$ are real numbers taken randomly in the interval $\{-1,1\}$ ). We compute the spectrum for a dimerized lattice for $\delta=0.5$ and average the results shown in Fig. 3(a) over 50 realizations. As it can be observed, only the dispersive part of the spectrum is perturbed in $\beta_{z}$ values (energies). The three edge states remain unaffected at their respective energies $\beta_{z}=0, \pm t_{3}$, for any degree of disorder. This shows the robustness of edge states against horizontal disorder, which is analytically proven in Appendix B. Since the energy of the left-side edge states depends on $t_{3}$ only, any perturbation to this coupling constant will produce a modification of this frequency and their robustness will be lost. However, the right-side edge state will persist as it is not affected by the coupling constant $t_{3}$.

Last but not least, we study the differences between our edge states and the well-known Tamm or defect states [21,22] appearing in trivial (nontopological) lattices [23]. To do this, we replace the first $t_{2}$ coupling coefficient by $t_{d}$, at the first unit cell of the lattice, as sketched in Fig. 3(b). Experimentally speaking, this is achieved by locally modifying the distance in between the first $B$ and $A$ sites (named $d_{d}$ ), as Fig. 3(c) clearly shows. We fabricate a set of lattices, varying the distance $d_{d}: 19,17,16,15,14,12 \mu \mathrm{m}$ (implying $t_{d}$ : $0.61,0.85,1.00,1.18,1.39,1.92 \mathrm{~cm}^{-1}$, respectively), considering a situation where the previously described edge states do not exist: $d_{1}=16 \mu \mathrm{m}$ and $d_{2}=14 \mu \mathrm{m} \rightarrow \delta \approx 1.4$. Then, using a characterization setup as the one shown in Fig. 2(c), we excite the $A$ sites at the first unit cell [see the yellow circle in Fig. 3(d1)]. Defect edge states appear due to the presence of an impurity or defect at the boundary. The inclusion of a coupling defect $t_{d}$ shows that these modes appear in the semi-infinite gap only, above and below the spectrum, and that they become well localized only for $t_{d} \gtrsim 1.8 t_{1}$ (see Appendix C). Figures 3(d1)-3(d3) show that for $t_{d} \leqslant t_{1}$ a localized FB pattern is observed with an increasing radiation tail to the right. For $t_{d}>t_{1}$ we observe a combined state [see in Figs. 3(d4)-3(d6)] where the FB profile disappears and a decaying profile emerges, which occupies sites on the main row mostly. This is certainly very different from the edge states described before, where $A$ sites are never excited.

\section{DISCUSSION}

One may wonder whether these states might be topological, a question which is also fueled by their robustness. A first aspect is that these edge states neither have the same energy (two of them appear at $\beta_{z}= \pm t_{3}$ and another one at $\beta_{z}=0$ ) nor appear in the same number at each edge. This contrasts with other known cases in one dimension (e.g., the SSH model $[9,10]$ ) where the topological states are midgap states, one at each edge, and where chiral symmetry pins their energy. Furthermore, there is another special property of our model resulting from its connectivity. Indeed, the lattice is bipartite, the sites making one frame (say $A$ and $C$ ) couple only to those in the second frame (sites $B$ ). The imbalance in the number of sites on each frame (unit chiral charge [11]) imposes one zero energy state per $k$ point $[11,24]$, i.e., a flat band, which for a finite system coexists with an edge state. All these elements hint that the reported edge states do not fall into the standard classification of topological states. However, our results suggest that their robustness against imperfections is comparable to that of the topological states in the SSH model.

In conclusion, we have theoretically and experimentally shown the appearance of unconventional edge states in quasione-dimensional dimerized Stub photonic lattices. Three exponentially localized edge states appear as the same transition regime as in the SSH model, but at three different energy values. These states are robust against horizontal disorder and are not originating due to effective defects at the edges. Our findings could open other opportunities for edge 

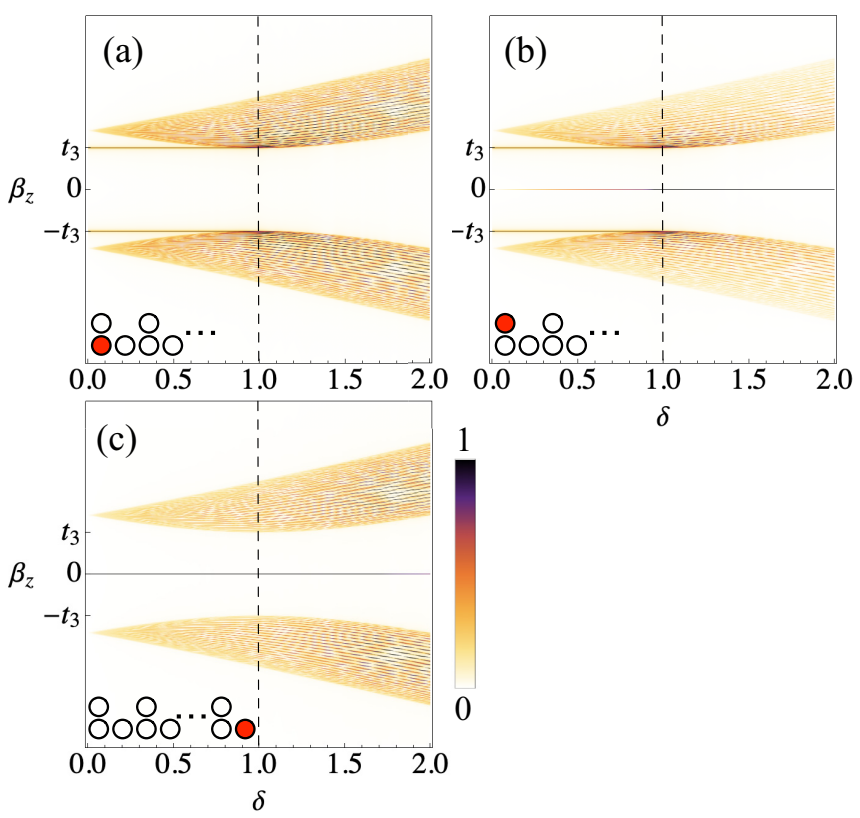

FIG. 4. The frequency spectrum vs parameter $\delta$ for (a) left- $B$, (b) left- $C$, and (c) right- $A$ input conditions, as indicated directly in the insets. The dashed line marks $\delta=1$ as the limit where edge modes exist. Simulations were performed considering 31 unit cells and a propagation length of $z_{\max }=1000$

localization at one-dimensional systems with controllable localization properties, robustness against disorder, and with a well-defined and controllable energy (see Appendix D).

\section{ACKNOWLEDGMENTS}

The authors acknowledge support from Millennium Science Initiative Program ICN17_012, FONDECYT Grants No. 1191205, No. 3190601, and No. 1211038, European Research Council grant EmergenTopo (865151), the H2020-FETFLAG project PhoQus (820392), the QUANTERA project Interpol (ANR-QUAN-0003-05), the French government through the Programme Investissement, d'Avenir (I-SITE ULNE/ANR16-IDEX-0004 ULNE) managed by the Agence Nationale de la Recherche, the Labex CEMPI (ANR-11-LABX-0007), and the CPER Photonics for Society P4S.

\section{APPENDIX A: FREQUENCY SPECTRUM FOR SINGLE-SITE EXCITATIONS}

By solving numerically Eqs. (1), up to a given distance $z_{\max }$, we obtain the light amplitudes $u_{n}(z)$ in the interval $\left\{0, z_{\max }\right\}$. Then, we compute a discrete Fourier transform along the propagation coordinate, for every waveguide, and get the information about the dynamically excited $\beta_{z}$ frequencies [25]. We integrate this information for all the lattice sites and generate a frequency spectrum diagram, which helps us to reveal the specific modes excited on a given dynamics. The results are shown in Fig. 4 for three different edge input conditions, as indicated in the insets of every figure.

Figure 4(a) shows the frequency spectrum for a $B$-edge excitation. For $\delta<1$, there is a strong excitation of edge states at frequencies $\beta_{z}= \pm t_{3}$, whereas for $\delta>1$ the excited

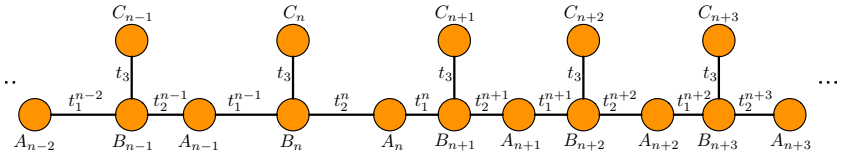

FIG. 5. Dimerized Stub lattice including disorder in coupling constants.

frequencies involve only bulk modes due to the absence of edge modes. In this case, there is no excitation of frequencies $\beta_{z}=0$, i.e., no flat-band modes are excited during propagation as shown in Fig. 2(c). Figure 4(b) shows the excitation of a $C$-edge site, which looks quite similar to the $B$-edge excitation; however, in this case, there are also frequencies at $\beta_{z}=0$, meaning that flat-band modes are also excited. Figure 4(c) shows the spectrum excited by an input condition at the right $A$-site boundary. We clearly observe that no frequencies associated with the edge modes $\beta_{z}= \pm t_{3}$ are present in the linear spectrum. FB and exponential modes at $\beta_{z}=0$ are indeed excited as well as extended bulk states. Considering these results, it is quite clear that edge modes at $\beta_{z}= \pm t_{3}$ are better excited by using a $B$-site input excitation, whereas hidden edge modes at $\beta_{z}=0$ are always superposed with FB states.

\section{APPENDIX B: EDGE STATE WITH DISORDER}

Let us assume that all horizontal couplings are different and depend on lattice positions as $t_{1}^{n}$ and $t_{2}^{n}$, as shown in Fig. 5 (this can be experimentally implemented by varying the distances in between the waveguides). In this case, we can write the stationary equations as follows,

$$
\begin{gathered}
\beta_{z} A_{n}=t_{2}^{n} B_{n}+t_{1}^{n} B_{n+1}, \\
\beta_{z} B_{n}=t_{2}^{n} A_{n}+t_{1}^{n-1} A_{n-1}+t_{3} C_{n}, \\
\beta_{z} C_{n}=t_{3} B_{n} .
\end{gathered}
$$

We find that the two edge states decaying from the left $B-C$ surface preserve their energy to $\beta_{z}= \pm t_{3}$, and have a modified exponential profile given by

$$
A_{n}=0, \quad B_{n}=B_{0} \delta_{n-1}, \quad C_{n}= \pm B_{n},
$$

where

$$
\delta_{n}=(-1)^{n} \prod_{i=1}^{n}\left(\frac{t_{2}^{i}}{t_{1}^{i}}\right) \delta_{0}, \quad \text { with } \delta_{0}=1
$$

If there is no disorder in the lattice, $t_{1}^{i}=t_{1}$ and $t_{2}^{i}=t_{2}$, and the expressions of the main text are recovered. However, when disorder is included, we observe that as soon as $t_{2}^{i} / t_{1}^{i}<1$, the edge states remain localized exponentially, i.e., the same condition for a homogenous lattice. We also observe that this is not a strict condition, and the requirement is that the product of all the ratios $t_{2}^{i} / t_{1}^{i}$ must be lower than 1 to fulfill the condition of exponential localization.

On the other hand, we check the effect of disorder at the right-hand $(A)$ edge state. Again, the frequency remains unchanged as $\beta_{z}=0$, and the profile modification can be 
written as follows,

$$
A_{n}=A_{N} \bar{\delta}_{n}, \quad B_{n}=C_{n}=0,
$$

where

$$
\bar{\delta}_{n}=(-1)^{N-n} \prod_{i=n}^{N-1}\left(\frac{t_{2}^{i+1}}{t_{1}^{i}}\right) \delta_{N}, \quad \text { with } \delta_{N}=1 .
$$

Again, if $t_{1}^{i}=t_{1}$ and $t_{2}^{i}=t_{2}$, expressions from the main text are recovered. When disorder on horizontal couplings is present, the condition for preserving the localization demands that ratios $t_{2}^{i+1} / t_{1}^{i}<1$, which is equivalent to the condition for the existence of edge localized modes on an ordered system.

In both cases, exponential localization is assured once the localized edge states are present on a full homogeneous lattice, due to the fact that $t_{2}<t_{1}$. In the main text, the disorder was included in the form $t_{1}^{i}=t_{1}+t_{0} \epsilon_{1}$ and $t_{2}^{i}=t_{2}+t_{0} \epsilon_{2}$, with $\epsilon_{i}$ real numbers from the interval $\{-1,1\}$. Therefore, as soon as the condition $t_{2}<t_{1}$ is fulfilled, $t_{2}^{i} / t_{1}^{i}<1$ and $t_{2}^{i+1} / t_{1}^{i}<1$ after averaging.

\section{APPENDIX C: TAMM MODES}

Tamm modes are known to exist when an effective defect is present at the edge of a given periodic system [21,23]. Therefore, we are interested in showing that the exponentially localized edge states found in this work do not originate from an effective defect, but from an originally dimerized $\mathrm{SSH}$ lattice which has been transformed into a Stub system. To study this, we consider the structure sketched in Fig. 3(b), where a coupling defect has been inserted at the first unit cell. Tamm states appear in pairs and are characterized by having a frequency outside the linear bands, one with a positive and one with a negative value, which is associated with an unstaggered and a staggered phase structure, respectively. First of all, in the absence of exponential edge states $(\delta>1)$, we only expect localization at the edge due to the excitation of FB modes or defect Tamm states. As an example, we numerically computed the whole linear spectrum for $\delta=1.4$ and analyzed the two Tamm states. The profile of both Tamm states is the same, apart from a phase; therefore, their localization properties are equal as shown in Fig. 6(a). There, we observe how the Tamm states are broad and delocalized for $t_{d} / t_{1} \lesssim 1.8$, while above this value the defect states start to increase their localization rapidly. In Fig. 6(b) we show three intensity profiles as examples. We observe that for different $t_{d}$ values a different profile is obtained in terms of localization, but also with the largest peak having been shifted from the bulk into the edge. For $t_{d} / t_{1}=1.85$ we see how the profile is broader and occupies several waveguides, while for $t_{d} / t_{1}=2.1$ and 3.0 the profile is well localized and decays from the surface into the bulk, as expected for an edge state. Interestingly, all sites in the main row are excited, which is a crucial contrast with respect to the exponential edge states shown in Figs. 1(d1)-1(d3), which only exist for $\delta<1$ and have some null sites in the main row. The experimental profiles shown in Fig. 3(d) are quite similar in shape and excited waveguides to the ones shown in
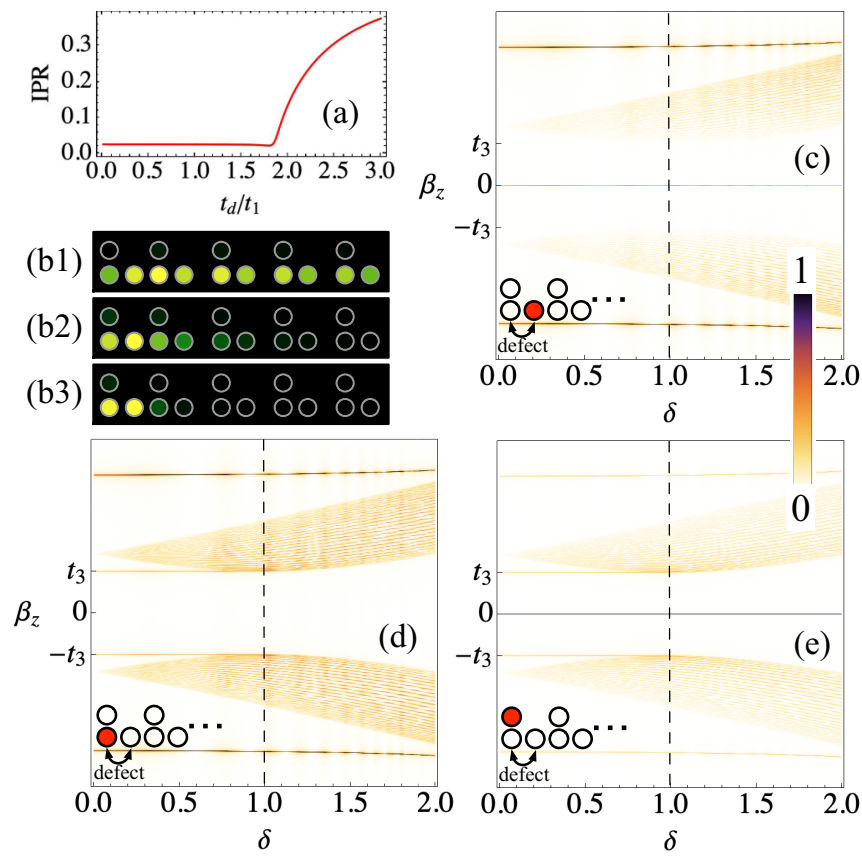

FIG. 6. (a) Inverse participation ratio (IPR) vs $t_{d} / t_{1}$ for both Tamm states, for $\delta=1.4$ and $t_{3}=1$. (b1)-(b3) Intensity profiles for Tamm states for $t_{d} / t_{1}=1.85,2.1$, and 3.0, for the same parameters used in (a) and using an avocado color scale. (c)-(e) Frequencies excited vs $\delta$, for $A-, B$-, or $C$-edge input conditions, as sketched in the insets. Simulations were performed considering 31 unit cells and a propagation length of $z_{\max }=1000$.

Fig. 6(b). This is confirmation that the exponential edge states found in this work do not have an origin in any effective defect at the edge, but are reminiscent of those in the SSH model obtained in the limit $t_{3} \rightarrow 0$.

We numerically computed the dynamically excited frequencies when varying the parameter $\delta$, considering different input conditions at the left edge (described in insets), for $t_{d}=3.0$ and $t_{3}=1$. For an $A$-edge excitation [see Fig. 6(c)], we observe that the edge states at $\beta_{z}= \pm t_{3}$ are not excited. However, FB modes and the two Tamm states are excited, the last ones corresponding to the two quasihorizontal lines above and below the bands. Therefore, in this case (equal to the experiment described in the main text) the localized profile observed above a given value of $t_{d}$ is originated only due to a defect, which in this case is a clear example of a Tamm state. When $B$ or $C$ sites are excited [see Figs. 6(d) and $6(\mathrm{e})]$, we observe that the edge states and Tamm modes are excited simultaneously, but FB states are excited only for a $C$ excitation. Note that the frequencies of the edge states are not perturbed by the presence of the Tamm modes, which shows their robustness against the inclusion of a local defect.

\section{APPENDIX D: PARAMETRIC STUDY OF $t_{3}$ COUPLING}

Edge states localized on a $B-C$ boundary have a frequency/energy that is set by the coupling among these two sites, $\beta_{z}= \pm t_{3}$. Therefore, the energy of these edge states can be modified at will by varying the coupling $t_{3}$. By computing 


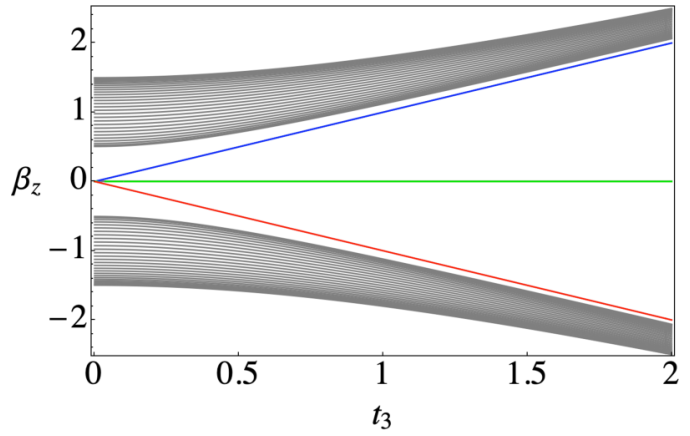

FIG. 7. Spectrum of a finite SSH-Stub lattice of 31 unit cells as a function of $t_{3}$ coupling when $\delta=0.5$. Gray lines correspond to the energies of bulk states. Blue, red, and green lines correspond to the energies of the edge states. the spectrum of a finite SSH-Stub lattice of 31 unit cells as a function of the $t_{3}$ coupling and considering $\delta=0.5$, we obtain the plot shown in Fig. 7. Gray lines present the energy evolution of the bulk states as a function of $t_{3}$, the blue and red lines correspond to the energy evolution of the left-boundary edge states, and the green line corresponds to the energy of the edge state localized on the right boundary. The amplitude profiles of the edge states are the ones shown in Figs. 1(d1)-1(d3). Since their spatial decay depends only on the parameter $\delta$, they exhibit the same spatial and phase pattern along the entire $t_{3}$ scan. The limit $t_{3}=0$ is actually the SSH model. Once the parameter $t_{3}$ is nonzero, two new edge states bifurcate from the left SSH edge state and the Zak phase is no longer quantized because inversion symmetry is broken [19]. As a consequence, there is no well-defined topological invariant in a SSH-Stub lattice.
[1] M. Z. Hasan and C. L. Kane, Colloquium: Topological insulators, Rev. Mod. Phys. 82, 3045 (2010).

[2] S. D. Huber, Topological mechanics, Nat. Phys. 12, 621 (2016).

[3] L. Lu, J. Joannopoulos, and M. Soljačić, Topological photonics, Nat. Photonics 8, 821 (2014).

[4] T. Ozawa, H. M. Price, A. Amo, N. Goldman, M. Hafezi, L. Lu, M. C. Rechtsman, D. Schuster, J. Simon, O. Zilberberg, and I. Carusotto, Topological photonics, Rev. Mod. Phys. 91, 015006 (2019).

[5] Y. Baum, T. Posske, I. C. Fulga, B. Trauzettel, and A. Stern, Coexisting Edge States and Gapless Bulk in Topological States of Matter, Phys. Rev. Lett. 114, 136801 (2015).

[6] L. E. F. Foa Torres, V. Dal Lago, and E. Suárez Morell, Crafting zero-bias one-way transport of charge and spin, Phys. Rev. B 93, 075438 (2016).

[7] M. S. Rudner, N. H. Lindner, E. Berg, and M. Levin, Anomalous Edge States and the Bulk-Edge Correspondence for Periodically Driven Two-Dimensional Systems, Phys. Rev. X 3, 031005 (2013).

[8] P. M. Perez-Piskunow, L. E. F. Foa Torres, and G. Usaj, Hierarchy of Floquet gaps and edge states for driven honeycomb lattices, Phys. Rev. A 91, 043625 (2015).

[9] W. P. Su, J. R. Schrieffer, and A. J. Heeger, Solitons in Polyacetylene, Phys. Rev. Lett. 42, 1698 (1979).

[10] J. K. Asbóth, L. Oroszlány, and A. Pályi, A Short Course on Topological Insulators, Lecture Notes in Physics Vol. 919 (Springer, Cham, 2016).

[11] M. Guzmán, D. Bartolo, and D. Carpentier, Geometry and topology tango in chiral materials, arXiv:2002.02850.

[12] D. Guzmán-Silva, C. Mejía-Cortés, M. A. Bandres, M. C. Rechtsman, S. Weimann, S. Nolte, M. Segev, A. Szameit and R. A. Vicencio, Experimental observation of bulk and edge transport in photonic Lieb lattices, New. J. Phys. 16, 063061 (2014).

[13] A. Yariv, Coupled-mode theory for guided-wave optics, IEEE J. Quantum Electron. 9, 919 (1973).
[14] R. A. Vicencio, Photonic flat band dynamics, Adv. Phys.: X 6, 1878057 (2021).

[15] J. He, Y.-X. Zhu, Y.-J. Wu, L.-F. Liu, Y. Liang, and S.-P. Kou, Zero modes around vacancies in topological insulators and topological superconductors on the honeycomb lattice with particle-hole symmetry, Phys. Rev. B 87, 075126 (2013).

[16] B. Real, C. Cantillano, D. López-González, A. Szameit, M. Aono, M. Naruse, S.-J. Kim, K. Wang, and R. A. Vicencio, Flat-band light dynamics in Stub photonic lattices, Sci. Rep. 7, 15085 (2017)

[17] P. Delplace, D. Ullmo, and G. Montambaux, Zak phase and the existence of edge states in graphene, Phys. Rev. B 84, 195452 (2011).

[18] A. Ramachandran, A. Andreanov, and S. Flach, Chiral flat bands: Existence, engineering, and stability, Phys. Rev. B 96, 161104(R) (2017).

[19] Y. Xiao, G. Ma, Z. Zhang, and C. T. Chan, Topological Subspace-Induced Bound State in the Continuum, Phys. Rev. Lett. 118, 166803 (2017).

[20] A. Szameit, D. Blömer, J. Burghoff, T. Schreiber, T. Pertsch, S. Nolte, A. Tünnermann, and F. Lederer, Discrete nonlinear localization in femtosecond laser written waveguides in fused silica, Opt. Express 13, 10552 (2005).

[21] I. Tamm, On the possible bound states of electrons on a crystal surface, Phys. Z. Sowjetunion 1, 733 (1932).

[22] H. Lüth, Solid Surfaces, Interfaces and Thin Films, Advanced Texts in Physics (Springer, Berlin, 2010).

[23] C. R. Rosberg, D. N. Neshev, W. Krolikowski, A. Mitchell, R. A. Vicencio, M. I. Molina, and Yu. S. Kivshar, Observation of Surface Gap Solitons in Semi-Infinite Waveguide Arrays, Phys. Rev. Lett. 97, 083901 (2006).

[24] D. Leykam and S. Flach, Perspective: Photonic flatbands, APL Photonics 3, 070901 (2018).

[25] U. Naether, A. J. Martínez, D. Guzmán-Silva, M. I. Molina, and R. A. Vicencio, Self-trapping transition in nonlinear cubic lattices, Phys. Rev. E 87, 062914 (2013). 